\title{
Differentiation between females of ostrich breeding trios based on egg weights
}

\author{
F. Essa ${ }^{1,2}$ and S.W.P. Cloete ${ }^{2 \#}$ \\ ${ }^{1}$ University of KwaZulu-Natal, Private Bag X01, Scottsville 3209, South Africa \\ ${ }^{2}$ Elsenburg Agricultural Development Centre, Private Bag X1, Elsenburg 7607, South Africa
}

\begin{abstract}
Data, collected during the 1997-1998 breeding season from 14 ostrich trios, were used to determine whether two females in a trio could be differentiated based on egg size. For eight of the trios a difference between egg weights could be discerned and eggs could be assigned to one or to the other female. An average egg weight of approximately $1.5 \mathrm{~kg}$ and an average chick weight of approximately $0.9 \mathrm{~kg}$ was recorded. Coefficients of variation ranged from $9.8 \%$ for chick weight to $10.7 \%$ for egg weight. Overall hatchability was $53 \%$. The repeatability of service sire observations for egg weight and chick weight were 0.32 and 0.28 respectively, whereas a higher repeatability of 0.82 and 0.67 , respectively, was recorded for these traits when based on individual females. Four of the eight trios showed differences in the assumed hatchability between the females. These results suggest a preference of certain males for specific females. This study shows that the process of differentiating between females and their eggs based on the egg weight is feasible and could contribute to better data structures for ostrich breeders during genetic evaluation.
\end{abstract}

Keywords: Pedigree, egg weight, differentiation, hatchability, repeatability

${ }^{\#}$ Corresponding author. E-mail: schalkc@elsenburg.com

\section{Introduction}

Within the ostrich industry there is a lack of basic pedigree and performance data recording, since about $80 \%$ of breeding birds are kept in colonies (usually at a male:female ratio of $6: 10$ ). It is impossible to obtain data for genetic analysis under such conditions. The industry thus lacks pedigree information linked to production data, which is the basis of livestock performance recording and evaluation schemes. Moreover, it is impossible to assess the reproduction potential of individual hens, which is known to be extremely variable. While breeding-pair mating systems enable accurate pedigree recording, the management practice of repeatedly pairing the same mates from year to year in the same breeding paddock means that potential random factors affecting performance will be confounded (Bunter, 2002). In order to improve data quality, reallocation of mates to different companions, and paddocks, from year to year can be done (Cloete et al., 2002; Bunter, 2002). Trio breeding, consisting of one male with two females, leads to an improved data structure but accurate recording of pedigrees remains a problem unless eggs from the two females can be differentiated on egg size or shell structure (Cloete et al., 1998; Bunter, 2002). High repeatability figures for egg weight from known breeding pairs reported by Cloete et al. (1998) and derived from variance ratios presented by Bunter (2002) support the reasoning that egg size could be used for differentiation. However, there is a suggestion that hatchability may be compromised under trio breeding conditions (Lambrechts et al., 2002). Conventional wisdom in the ostrich industry suggests that all males are not necessarily compatible with all females, which could lead to lower hatchability under trio mating circumstances. This study investigates production records of trios to determine whether different females can be differentiated according to egg weights using historical data. Simultaneously hatchability was compared for the two females comprising a trio in cases where it was possible to differentiate on the basis of egg size.

\section{Materials and Methods}

Data were obtained from 14 trios of the 1997-1998 breeding season. These trios were maintained at the Klein Karoo Agricultural Development Centre, near Oudtshoorn, South Africa. For each trio, data were first sorted according to egg production date. Then egg weight was plotted against production date to determine if the eggs of the two females in a trio could be differentiated, based on egg weight. Wherever this was possible with a trio, the eggs of higher weight were assigned to one female and the eggs of lower weight to the other female. Nine eggs were excluded from the study as they were broken prior to weighing, and thus could not be assigned to a specific female.

The ASREML program (Gilmour et al., 1999) was used to fit random effects of service sire initially, and then of dam to get an indication of the repeatability of egg weight and chick weight. Variance 
components derived in this way were used to obtain repeatability estimates based on either sires or dams. It was reasoned that correspondence of these estimates with the literature would provide an indication of the success achieved with the allocation of eggs to individual females.

Hatchability was calculated using only the trios that could be differentiated. A chi-squared two-bytwo contingency table was used to test if there were significant differences in hatchability between the two females in a trio (Siegel, 1956).

\section{Results and Discussion}

It was possible to discern between females based on egg weight differences for eight out of the 14 trios (Figure 1). In cases with very similar egg weights, it was not possible to make a reliable distinction between females in the trio (Figure 2).

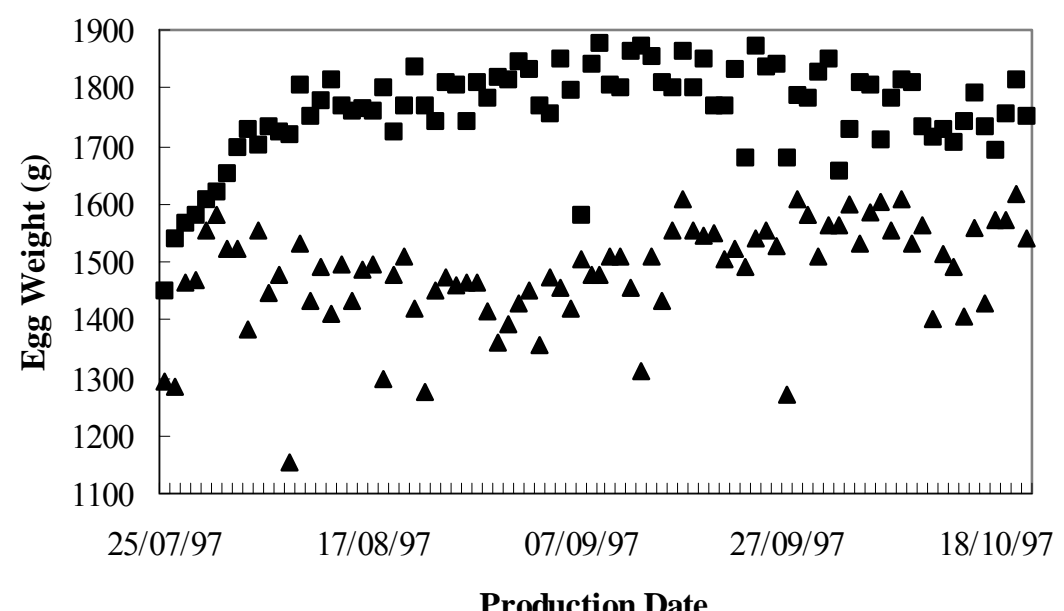

Figure 1 Egg weights plotted against production date for a trio where clear differences were evident between the two females, denoted by squares and triangles respectively

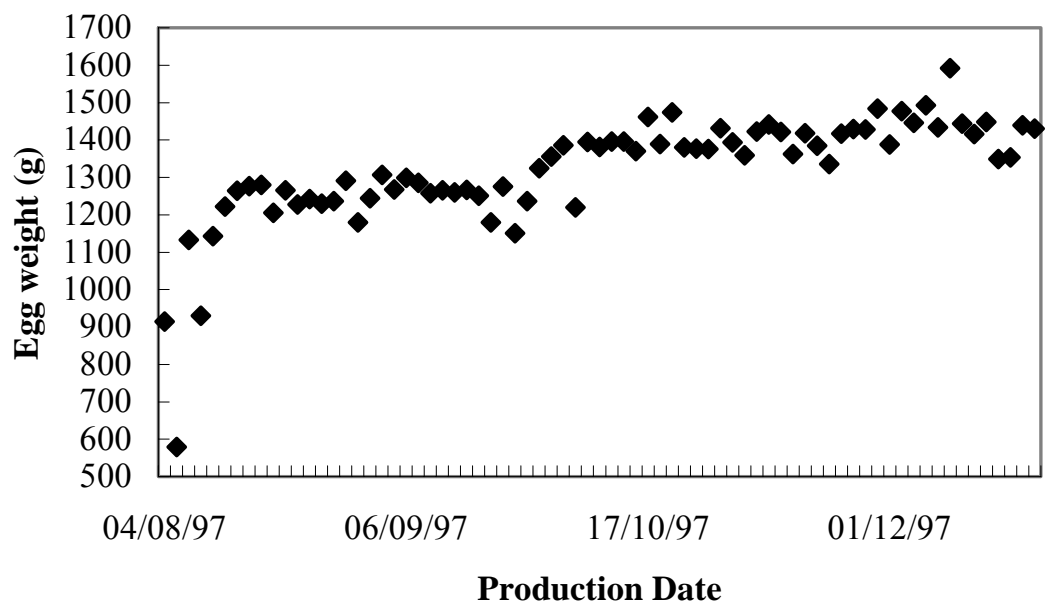

Figure 2 Egg weights plotted against production date for a trio where it was impossible to discern between the two females

Mean egg and chick weights were approximately $1.5 \mathrm{~kg}$ and $0.9 \mathrm{~kg}$ respectively (Table 1). Coefficients of variation ranged from $9.8 \%$ for chick weight to $10.7 \%$ for egg weight. These results are consistent with previous reports (Cloete et al., 1998; Bunter, 2002). An overall hatchability of approximately $53 \%$ was computed from figures in Table 1, which is slightly higher than the average of $46 \%$ reported by Cloete et al. (1998). The repeatability based on service sire amounted to approximately 0.30 for both traits. This figure is substantially lower than previous figures ranging from 0.61 to 0.87 for egg weight 
and from 0.56 to 0.73 for chick weight (Cloete et al., 1998; Bunter, 2002). Estimates within these ranges ( 0.82 and 0.67 respectively) were obtained when the random source of variation was based on individual females.

In half of the trios under investigation, no difference could be discerned between the hatchability of eggs from the two females (Table 2). However, in the other four trios, there were indications of differences between the two members $(\mathrm{P}=0.11$ to $\mathrm{P}<0.01)$. This indirect evidence support arguments in favour of varying levels of preference of certain males for specific females. These results also give substance to the reduction in hatchability of trios reported by Lambrechts et al. (2002).

Table 1 Descriptive statistics for egg and chick weights

\begin{tabular}{lcc}
\hline Parameter & Egg weight $(\mathrm{g})$ & Chick weight $(\mathrm{g})$ \\
\hline Number of observations & 791 & 419 \\
Overall mean & 1502 & 892 \\
Standard deviation & 160 & 87 \\
Repeatability & & \\
Based on the male variance & $0.32 \pm 0.12$ & $0.28 \pm 0.11$ \\
Based on the female variance & $0.82 \pm 0.06$ & $0.67 \pm 0.09$ \\
\hline
\end{tabular}

Table 2 Egg numbers, hatchability figures and statistical information for individual females per trio

\begin{tabular}{lccccc}
\hline Sire ID & Hypothesized Dam ID & Hatchability (Dam A) & Hatchability (Dam B) & $\chi^{2}$ & Significance \\
\hline 56 & $149 / 150$ & $23 / 65=0.35$ & $23 / 56=0.41$ & 0.207 & $\mathrm{P}>0.25$ \\
93 & $147 / 148$ & $53 / 84=0.63$ & $54 / 84=0.64$ & 0.000 & $\mathrm{P}>0.25$ \\
101 & $151 / 152$ & $42 / 68=0.62$ & $5 / 51=0.10$ & 30.788 & $\mathrm{P}<0.01$ \\
113 & $177 / 178$ & $42 / 48=0.88$ & $11 / 19=0.58$ & 5.538 & $\mathrm{P}<0.05$ \\
131 & $179 / 180$ & $40 / 57=0.70$ & $12 / 22=0.55$ & 1.099 & $\mathrm{P}>0.25$ \\
159 & $159 / 160$ & $16 / 30=0.53$ & $36 / 49=0.73$ & 2.518 & $\mathrm{P}=0.11$ \\
162 & $165 / 166$ & $24 / 63=0.38$ & $3 / 21=0.14$ & 3.075 & $\mathrm{P}=0.08$ \\
168 & $173 / 174$ & $34 / 57=0.60$ & $10 / 17=0.59$ & 0.049 & $\mathrm{P}>0.25$ \\
\hline
\end{tabular}

\section{Conclusions}

The differentiating between females in a trio based on egg size seems feasible, provided that there is some indication of a weight difference between the eggs of those females involved. The method should be particularly effective if previous knowledge of egg size, -shape, -colour and -structure of individual females could be obtained from birds previously subjected to pair breeding. This could contribute to better data structures without resorting to more expensive techniques, for example, DNA fingerprinting, which is being developed. The latter procedures are however required for verification of the accuracy of this technique.

\section{References}

Bunter, K.L., 2002. The genetic analysis of reproduction and production traits recorded for farmed ostriches (Struthio camelus). PhD dissertation, University of New England, Australia.

Cloete, S.W.P., Bunter, K.L. \& van Schalkwyk, S.J., 2002. Progress towards a scientific breeding strategy for ostriches. Proc. $7^{\text {th }}$ World Congr. Gen. Appl. Livest. Prod. 30, 561-568. Montpellier, France.

Cloete, S.W.P., van Schalkwyk, S.J. \& Brand, Z., 1998. Ostrich breeding - progress towards a scientifically based strategy. Proc. $2^{\text {nd }}$ International Ratite Congr., Ed. F.W. Huchzermeyer, Oudtshoorn, South Africa, 21-25 September 1998. pp. 55-62.

Gilmour, A.R., Cullis, B.R., Welham, S.J. \& Thompson, R., 1999. ASREML - Reference manual. NSW Agriculture Biometric Bull. No. 3. NSW Agriculture, Orange Agricultural Institute, Forest Road, Orange 2800, NSW, Australia.

Lambrechts, H., Swart, D., Cloete, S.W.P., van Schalkwyk, S.J. \& Greyling, J.P.C., 2002. Egg production and fertility of breeding ostriches as influenced by stocking density and male : female ratio. Proc. World Ostrich Congr., Ed. Horbanczuk, J.O., Warsaw, Poland, 26-29 September 2002. pp. 211-213.

Siegel, S., 1956. Nonparametric statistics for the behavioural sciences. McGraw-Hill Book Co. (Inc), New York. 